\title{
Clinical and functional outcomes of tarsal coalition resection to correct rigid flat foot
}

\author{
Rodrigo Guimarães Huyer $\mathbb{1 D}^{\mathbb{D}}$, Mário Sérgio Paulillo Cillo ${ }^{\circledR}$, Carlos Daniel Cândido Castro Filho' ${ }^{\mathbb{D}}$, Hallan Douglas Bertelli'(D), \\ Marcelo Morelli Girondo ${ }^{(\mathbb{D})}$, Armando Bortolatto Neto ${ }^{1}$ (D) \\ 1. Orthopedics and Traumatology Departament PUC-Campinas, Campinas, SP, Brazil.
}

\begin{abstract}
Objective: This study used the AOFAS score to assess the clinical functional results of patients who underwent tarsal coalition resection. Methods: This was a retrospective case series of patients who underwent tarsal coalition resection to correct rigid flat foot. Clinical and functional assessment was performed with the AOFAS score before and 6 months after surgical treatment. Descriptive analysis was performed for 7 patients (11 operated feet) using measurements of position and dispersion (mean, standard deviation, minimum, median and maximum value) for continuous variables and frequency tables (absolute and relative) for categorical variables.

Results: The mean patient age was 10 years, 7 months, and the majority (71.43\%) were male. The most affected joint was the calcaneonavicular. The right side was affected in $54.55 \%$ of the cases. The most frequent type of coalition was osseous ( $81.82 \%$ of the cases). The mean pre- and postoperative AOFAS scores were 32.7 and 70.2 points, respectively, which was a significant increase.

Conclusion: The increased scores after coalition resection was considered the main change between the two assessments. Thus, it can be concluded that in rigid flat feet without severe hind- or forefoot deformities for which conservative treatment failed, bar resection should be the surgical procedure of choice.
\end{abstract}

Level of Evidence IV; Therapeutic Studies; Case Series.

Keywords: Tarsal coalition; Tarsal bones; Flatfoot; Subtalar joint.

\section{Introduction}

Tarsal coalition, a bone pathology that occurs in less than $1 \%$ of the population ${ }^{(1)}$, is characterized as a bony, cartilaginous or fibrous connection between two or more bones of the hind-and/or midfoot. It is the main cause of rigid flat foot in children. Although many patients are asymptomatic, others complain of foot pain, functional limitations, and ankle sprains $^{(2-4)}$. Symptom onset is related to the ossification process: ossification occurs in the calcaneonavicular coalition and the talocalcaneal joint at 8-12 and 12-16 years of age, respectively ${ }^{(5)}$. Clinical diagnosis can be complemented by radiographic examinations, which should include an oblique view. Magnetic resonance imaging and computed tomography can be useful complementary methods in the diagnostic process. Conservative treatment initially involves behavioral and lifestyle recommendations, non-steroidal anti-inflamma- tory drugs, and insoles ${ }^{(6,7)}$. Immobilization with or without a cast is another conservative treatment option. Surgical treatment includes bar resection and, when the coalition compromises a large joint surface, arthrodesis.

Surgical treatment is recommended for symptomatic tarsal coalition, since it can more efficiently restore normal foot function ${ }^{(8)}$ and avoids the unsatisfactory results of immobilization ${ }^{(3)}$, in addition to preventing sequelae by eliminating the cause of the pathology ${ }^{(8,9)}$. However, some authors recommend conservative treatment for symptomatic coalitions, whether calcaneonavicular ${ }^{(10-13)}$ or talocalcanea|(7-8,10-12,14-16).

The present study assessed the functional clinical results of resecting tarsal coalition in patients with symptomatic rigid flat foot, comparing American Orthopedic Foot and Ankle Society (AOFAS) scores before and after the procedure ${ }^{(17)}$.
Study performed at the Orthopedics and Traumatology Departament PUC-Campinas, Campinas, SP, Brazil.

Correspondence: Marcelo Morelli Girondo. Rua Alberto Cerqueira Lima, 29, Apto. 171B, Taquaral - 13076-010, Campinas, SP, Brazil. E-mail: girondomarcelo@gmail.com. Conflicts of interest: none. Source of funding: none. Date received: May 20, 2021. Date accepted: July 05, 2021. Online: August 31, 2021.
How to cite this article: Huyer RG, Cillo MSP, Castro Filho CDC, Bertelli HD, Girondo MM, Bortolatto

Neto A. Clinical and functional outcomes of tarsal coalition resection to correct rigid flat foot. J Foot Ankle. 2021;15(2):115-9.

\section{c) (P) $\$$}




\section{Methods}

This retrospective clinical study, which was approved by the institutional ethics committee, included 7 patients (11 feet) who underwent tarsal coalition resection to treat rigid flat foot. The following data were collected from our institution's database and stored for further evaluation: age at the time of surgery, sex, type of coalition (determined at the time of surgery: fibrous, cartilaginous, or osseous), affected joint (talocalcaneal or calcaneonavicular), complementary diagnostic methods in addition to physical examination, and the symptoms presented. Valente's classification for flat foot (to grade 3) was used to indicate tarsal coalition resection. All patients had moderate deformities and showed no signs of joint degeneration or arthrosis.

The AOFAS scoring system was developed as a standardized method for clinical and functional assessment of different parts of the foot, enabling better pathological analysis and therapeutic planning. The ankle and hindfoot scale, which totals 100 points, consists of nine items in three categories: pain (40 points), functional aspects (50 points) and alignment (10 points). The AOFAS scale was applied during the preoperative period and again 6 months after tarsal coalition resection. The results were compared using statistical methods. All patients were followed for at least 12 months.

The patients and feet were descriptively analyzed by measures of the central position and dispersion (mean, standard deviation, and minimum, median, and maximum values) for continuous variables and frequency tables (absolute and relative) for categorical variables.

Generalized estimating equations were used to study AOFAS scores over time. The estimates were calculated using maximum likelihood models for control, without assuming independence between subjects or within-subject variability. The values were transformed into ranks due to their non-normal distribution.

A significance level of $5 \%$ was used for the statistical tests.

\section{Results}

The patients were classified according to age, sex, type of coalition, affected joint, laterality, and complementary diagnostic methods.

The patients' mean age was 10.7 years and $71.43 \%$ were male. The most frequent complementary diagnostic method was radiography (57.14\% of the cases), followed by an association of radiography and computed tomography (42.86\%).

Of the orthopedic lesions $54.55 \%$ were in the right lower limb and $45.45 \%$ were in the left lower limb. Osseous coalition was the most frequent type (81.82\% of the patients), followed by cartilaginous (18.18\%); none of the coalitions were fibrous. The calcaneonavicular and talocalcaneal joints were affected in $54.55 \%$ and $45.45 \%$ of the cases, respectively (Table 1).

The mean pre- and postoperative AOFAS scores were 32.7 and 70.2, respectively, which was a significant increase. We consider this the main effect of the change between the two assessments (Table 2 and Figure 1).
The relationship between sex, age, and affected joint in the pre- and postoperative periods was also analyzed. Among female patients, the mean pre- and postoperative AOFAS scores were 23.0 and 78.5 points, respectively, while for males, they were 34.9 and 68.3 points, respectively. There was no significant difference in pre/post score change between the sexes (Table 3 and Figure 2).

Table 1. General descriptive analysis

\begin{tabular}{|c|c|c|c|}
\hline \multicolumn{4}{|c|}{ Age } \\
\hline Mean & SD & Min. & Max. \\
\hline 10.7 & 2.4 & 7.0 & 14.0 \\
\hline \multicolumn{4}{|c|}{ Sex } \\
\hline \multicolumn{4}{|c|}{ Cumulative } \\
\hline Sex & Frequency & Percent & Frequency \\
\hline Female & 2 & 28.57 & 2 \\
\hline Male & 5 & 71.43 & 7 \\
\hline \multicolumn{4}{|c|}{ Type of coalition (fibrous, cartilaginous, osseous) } \\
\hline \multicolumn{4}{|c|}{ Cumulative } \\
\hline Coalition & Frequency & Percent & Frequency \\
\hline Cartilaginous & 2 & 18.18 & 2 \\
\hline Osseous & 9 & 81.82 & 11 \\
\hline \multicolumn{4}{|c|}{ Affected joint (TC, CN) } \\
\hline \multicolumn{4}{|c|}{ Cumulative } \\
\hline JOINT & Frequency & Percent & Frequency \\
\hline $\mathrm{CN}$ & 6 & 54.55 & 6 \\
\hline $\mathrm{TC}$ & 5 & 45.45 & 11 \\
\hline \multicolumn{4}{|c|}{ Complementary diagnostic method } \\
\hline \multicolumn{4}{|c|}{ Cumulative } \\
\hline Diagnostic method & Frequency & Percent & Frequency \\
\hline Radiography & 4 & 57.14 & 4 \\
\hline Radiography + CT & 3 & 42.86 & 7 \\
\hline \multicolumn{4}{|c|}{ Laterality } \\
\hline \multicolumn{4}{|c|}{ Cumulative } \\
\hline Laterality & Frequency & Percent & Frequency \\
\hline Right foot & 6 & 54.55 & 6 \\
\hline Left foot & 5 & 45.45 & 11 \\
\hline
\end{tabular}

CN: calcaneonavicular; CT: computed tomography; TC: talocalcaneal.

Table 2. Descriptive analysis and comparison of AOFAS scores between assessments

\begin{tabular}{|c|c|c|c|c|c|c|}
\hline Variable & $\mathbf{N}$ & Mean & SD & Min. & Median & Max. \\
\hline AOFAS_PRE & 11 & 32.7 & 19.1 & 10.0 & 38.0 & 55.0 \\
\hline AOFAS_POST & 11 & 70.2 & 13.2 & 51.0 & 77.0 & 83.0 \\
\hline
\end{tabular}




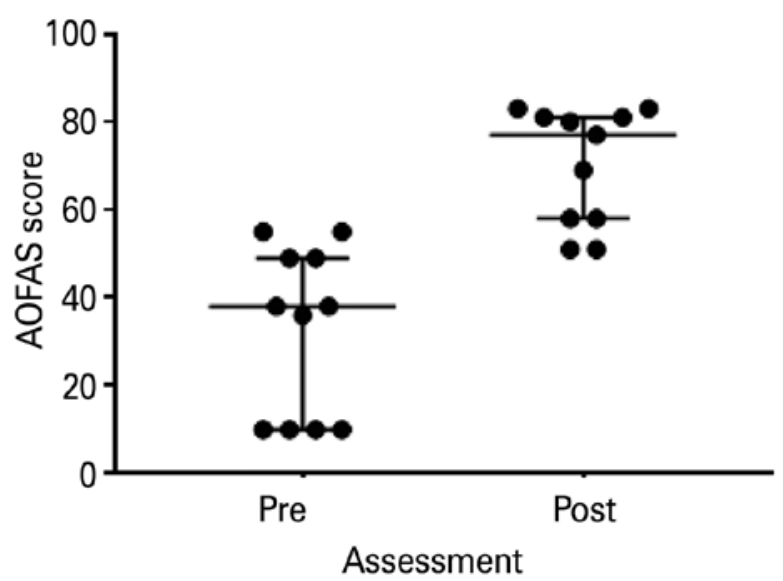

Figure 1. AOFAS score dispersion in each assessment. The bars represent the median and the interquartile interval. There was significant difference between the assessments in general $(p=0.0259$ EEG).

Table 3. Descriptive analysis and comparison of AOFAS scores between the sexes

\begin{tabular}{lccccccc} 
Sex & Variable & N & Mean & SD & Min. & Median & Max. \\
Fem. & AOFAS_PRE & 2 & 23.0 & 18.4 & 10.0 & 23.0 & 36.0 \\
& AOFAS_POST & 2 & 78.5 & & 77.0 & 78.5 & 80.0 \\
\multirow{2}{*}{ Male } & AOFAS_PRE & 9 & 34.9 & 19.6 & 10.0 & 38.0 & 55.0 \\
& AOFAS_POST & 9 & 68.3 & & 51.0 & 69.0 & 83.0 \\
\hline
\end{tabular}

$\mathrm{P}=0.3863$ (GEE). There was no significant difference in pre/post score change between the sexes.

The operated side was considered as a control factor (repeated measure with missing data), and pre/post change and sex were considered effects.

GEE: generalized estimating equation.

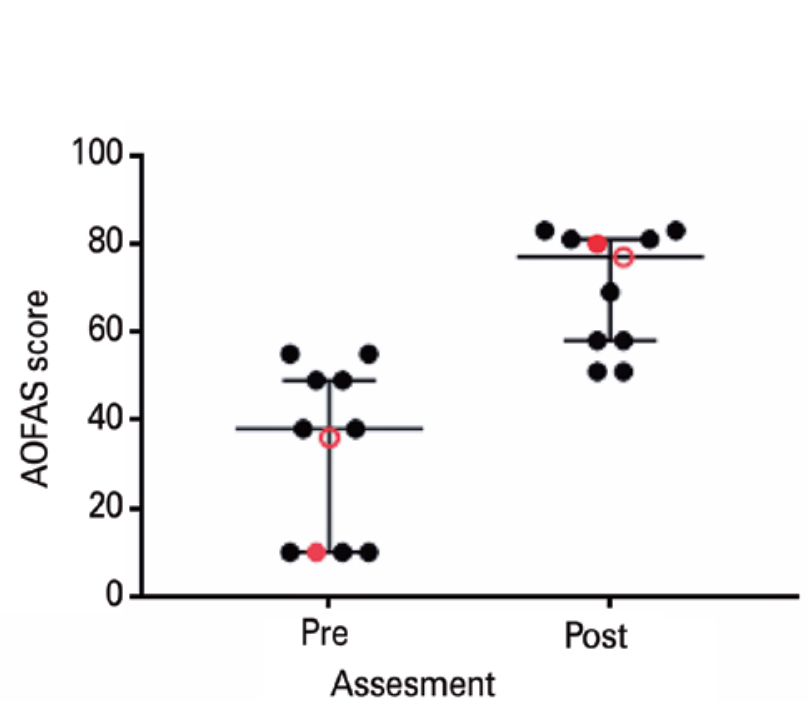

Figure 2. Dispersion of the AOFAS score in each assessment. The bars represent the medians and interquartile intervals in general. The red marks represent women. There was no significant difference between genders in each assessment $(p=0.3863$ EEG).
The mean pre- and postoperative AOFAS scores of patients diagnosed with a talocalcaneal tarsal coalition were 36.8 and 68.8 , respectively. In patients whose calcaneonavicular joint was affected, the mean pre- and postoperative scores were 29.3 and 71.3, respectively. There was no significant difference in pre/post score change between the affected joints (Table 4 and Figure 3). There was also no significant relationship between age and pre/post score change (Table 5).

Table 4. Descriptive analysis and comparison of the AOFAS scores between the affected joints

\begin{tabular}{|c|c|c|c|c|c|c|c|}
\hline Joint & Variable & N & Mean & SD & Min. & Median & Max. \\
\hline \multirow[t]{2}{*}{$\mathrm{CN}$} & AOFAS_PRE & 6 & 29.3 & 22.3 & 10.0 & 23.0 & 55.0 \\
\hline & AOFAS_POST & 6 & 71.3 & 11.5 & 58.0 & 73.0 & 83.0 \\
\hline \multirow[t]{2}{*}{ TC } & AOFAS_PRE & 5 & 36.8 & 16.0 & 10.0 & 38.0 & 49.0 \\
\hline & AOFAS_POST & 5 & 68.8 & 16.3 & 51.0 & 80.0 & 81.0 \\
\hline
\end{tabular}

CN: calcaneonavicular; GEE generalized estimating equation; TC: talocalcaneal.

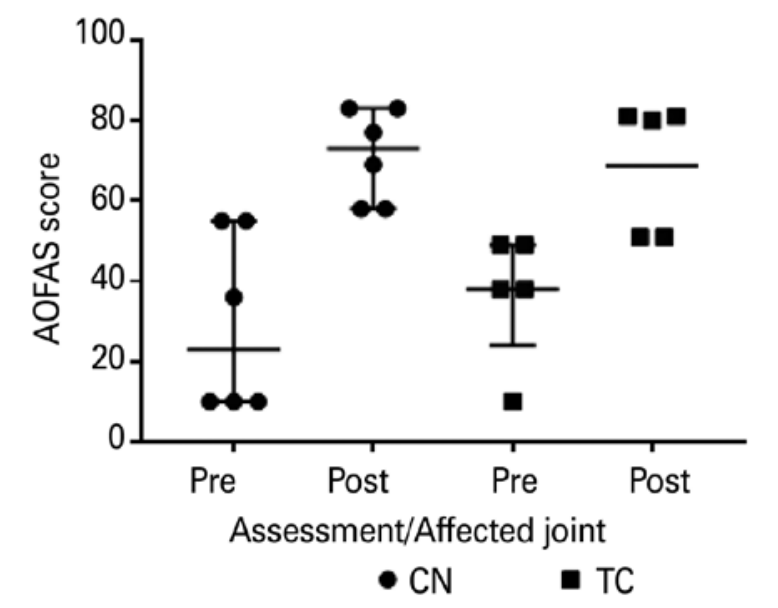

Figure 3. Dispersion of AOFAS score in each time and affected joint. The bars represent the median and interquartile intervals in each of the affected joints. There was no significant difference between de assesments for each of the affected joints. ( $p=0.6537$ EEG).

Table 5. GEE results on the relationship between age and AOFAS score

$\mathrm{P}=0.0592$ (GEE). There was no significant relationship between pre/post score change and age.

The operated side was considered as a control factor (repeated measure with missing data), and pre/post change and age were considered effects. 


\section{Discussion}

The main goal of tarsal coalition treatment is symptom relief and a biomechanically stable foot that does not cause functional impairment. According to the literature, as well as our results, when patients are managed according to a treatment flowchart, good results almost always occur.

In a recent study on conservative treatment for tarsal coalitions, Shirley et al. ${ }^{(18)}$ found that it can have a positive effect on pain, as well as the prevention or postponement of surgical treatment for symptomatic cases. Although the results of conservative treatment cannot be compared with those of our study, from a treatment point of view, conservative treatment should not be ruled out and may produce good results. In the patients in our study, surgery was indicated because conservative treatment failed. Therefore, a good relationship with the patient's parents and making sure they understand the treatment process is an important part of the approach at our clinic. As pain complaints increase in frequency and intensity, the treatment strategy should be changed to bar resection.

Scranton ${ }^{(19)}$ re-evaluated 14 patients (23 tarsal coalitions) who had been treated conservatively after a mean of 3.9 years (2.2 to 9.5 years) of treatment. Five feet in 3 patients were asymptomatic after plaster cast immobilization, 4 feet underwent triple arthrodesis, and 14 feet underwent coalition resection after immobilization failed to resolve the symptoms. As in our study, non-response to conservative treatment was an indication for resection. In addition, the author considered a coalition smaller than half of the area of the affected joint and no arthrosis in the affected joint (mainly talocalcaneal coalition) as indications for surgery. Considering all forms of treatment, the results were good in 13 feet and satisfactory in the other 10. For the present study, we chose bar resection for all patients, and the surgical results are based on an early appropriate diagnosis, considering patient age and light clinical deformity (hindfoot valgus and forefoot abduction). In coalition cases involving severe deformities of the forefoot and hindfoot, calcaneal osteotomies are a more common treatment. Our group views isolated subtalar arthrodesis and double or triple joint remodeling as exceptional and salvage surgical treatments.

Also using bar resection, Kumar et al. ${ }^{(14)}$ found excellent results in 8 feet, good results in 8 feet, and poor results in 1 foot (due to recurrent tarsal coalition). The types of coalition identified in the preoperative exams were confirmed during surgery, and the type of coalition did not influence the surgical outcome. These results demonstrate the effectiveness of tarsal coalition resection, especially after conservative treatment fails.
Gonzalez et al. ${ }^{(15)}$ obtained excellent or good results in $77 \%$ of patients they treated with bar resection. In 3 of the patients who reported unsatisfactory results during follow-up, symptom improvement occurred by the end of follow-up and their final results was considered good. The best results were observed in patients who had a cartilage coalition and were under 16 years of age at the time of surgery. In our stu$\mathrm{dy}$, osseous coalition was the most frequent type $(81.82 \%$ of patients), followed by cartilaginous (18.18\%); there were no fibrous coalitions. There were no significant differences between coalition types in AOFAS score improvement. As in the literature, the most affected joint in our study was the calcaneonavicular ( $54.55 \%$ of the cases), followed by the talocalcaneal (45.45\%).

Takakura et al. ${ }^{(20)}$ compared surgical and conservative treatment in 29 patients (36 feet) whose tarsal coalition was diagnosed using computed tomography as a complementary method. A total of 33 tarsal coalition resections and 3 arthrodeses were performed. The follow-up time averaged 5.3 years (2.25 to 11.2 years). The resection results were excellent in 24 feet and good in 7, with 2 treatment failures. The arthrodesis results were good in all 3 feet.

In 2009 and 2016, Hamel(21,22) evaluated 24 resections of talocalcaneal coalitions in 22 patients. After 21.2 months of follow-up, complete symptom remission occurred in $17 \mathrm{pa}-$ tients. Five others still had pain, although it had improved, and 2 of these were lost to follow-up.

In our study, patients' mean age was 10.7 years and $71.43 \%$ were male. Our mean follow-up time was 2.44 years (1.333.58). The most frequent preoperative complementary diagnostic method was isolated radiography (57.14\% of the cases), followed by an association of radiography and computed tomography (42.86\%).

Our small sample size can be considered a major limiting factor.

Due to the mean increase in AOFAS score between the preand postoperative periods, we can conclude that the results were good. The significant increase in postoperative scores must be considered an effect of the surgery.

\section{Conclusion}

As in the other cited studies, we found that bar resection should be the surgical procedure of choice in rigid flat feet without severe hindfoot or forefoot deformities that are refractory to conservative treatment.

Authors' contributions: Each author contributed individually and significantly to the development of this article: RGH *(https://orcid.org/OOOO-OOO33951-8408) Conceived and planned the activities that led to the study, bibliographic review, participated in the review process and approved the final version; MSPC *(https://orcid.org/0000-0002-0758-2547) Conceived and planned the activities that led to the study, bibliographic review, participated in the review process and approved the final version; CDCCF *(https://orcid.org/O000-0003-3522-1076) Performed the surgeries, data collection and approved the final version; HDB *( https://orcid.org/O000-0002-1901-3309) Performed the surgeries, data collection and approved the final version; MMG *(https://orcid.org/0000-0001-7187-4774) Interpreted the results of the study, participated in the review process, data collection and formatting of the article; $A B N^{*}$ (https://orcid.org/0000-0002-2442-0427) Interpreted the results of the study, participated in the review process, data collection and formatting of the article. All authors read and approved the final manuscript. *ORCID (Open Researcher and Contributor ID) iD). 


\section{References}

1. Stormont DM, Peterson HA. The relative incidence of tarsal coalition. Clin Orthop Relat Res. 1983;(181):28-36.

2. Gantsoudes GD, Roocroft JH, Mubarak SJ. Treatment of talocalcaneal coalitions. J Pediatr Orthop 2012;32(3):301-7.

3. Mubarak SJ, Patel PN, Upasani VV, Moor MA, Wenger DR. Calcaneonavicular coalition: treatment by excision and fat graft. J Pediatr Orthop. 2009;29(5):418-26.

4. Snyder RB, Lipscomb AB, Johnston RK. The relationship of tarsal coalitions to ankle sprains in athletes. Am J Sports Med. 1981; 9(5):313-7.

5. Downey MS. Tarsal coalition. In: Banks AS, Downey MS, Martin DE and Miller SJ, editors. McGlamry's comprehensive textbook of foot and ankle surgery. 3rd ed. Philadelphia, PA: Lippincott Williams \& Wilkins, 2001. p. 993-1031.

6. Blakemore LC, Cooperman DR, Thompson GH. The rigid flatfoot. Tarsal coalitions. Clin Podiatr Med Surg. 2000;17(3):531-55.

7. Mosca VS. Subtalar coalition in pediatrics. Foot Ankle Clin. 2015; 20(2):265-81.

8. Jayakumar S, Cowell HR. Rigid flatfoot. Clin Orthop Relat Res. 1977;(122):77-84.

9. Murphy JS, Mubarak SJ. Talocalcanealal Coalitions. Foot Ankle Clin. 2015;20(4):681-91.

10. Zaw H, Calder JD. Tarsal coalitions. Foot Ankle Clin. 2010;15(2):349-64.

11. Lemley F, Berlet G, Hill K, Philbin T, Isaac B, Lee T. Current concepts review: Tarsal coalition. Foot Ankle Int. 2006;27(12):1163-9.

12. Vincent KA. Tarsal coalition and painful flatfoot. J Am Acad Orthop Surg. 1998;6(5):274-81.
13. Swensen SJ, Otsuka NY. Tarsal Coalitions--Calcaneonavicular Coalitions. Foot Ankle Clin. 2015;20(4):669-79.

14. Kumar SJ, Guille JT, Lee MS, Couto JC. Osseous and non-osseous coalition of the middle facet of the talocalcaneal joint. $J$ Bone Joint Surg Am. 1992;74(4):529-35.

15. Gonzalez P, Kumar SJ. Calcaneonavicular coalition treated by resection and interposition of the extensor digitorum brevis muscle. J Bone Joint Surg Am. 1990;72(1):71-7.

16. Cowell HR. Talocalcanealal coalition and new causes of peroneal spastic flatfoot. Clin Orthop Relat Res. 1972;85:16-22.

17. Rodrigues RC, Masiero D, Mizusaki JM, Imoto AM, Peccin MS Cohen $\mathrm{M}$, et al. Translation, cultural adaptation and validity of the "American Orthopaedic Foot and Ankle Society (AOFAS) Ankle-Hindfoot Scale. Acta Ortop Bras. 2008;16(2):107-11.

18. Shirley E, Gheorghe R, Neal KM. Results of Nonoperative Treatment for Symptomatic Tarsal Coalitions. Cureus. 2018;10(7):e2944.

19. Scranton PE Jr. Treatment of symptomatic talocalcaneal coalition. J Bone Joint Surg Am. 1987;69(4):533-9.

20. Takakura Y, Sugimoto K, Tanaka Y, Tamai S. Symptomatic talocalcaneal coalition. Its clinical significance and treatment. Clin Orthop Relat Res. 1991;(269):249-56.

21. Hamel J. [Resection of talocalcaneal coalition in children and adolescents without and with osteotomy of the calcaneus]. Oper Orthop Traumatol. 2009;21(2):180-92.

22. Hamel J, Nell M, Rist C. [Surgical treatment of talocalcaneal coalition: Experience with 80 cases of pediatric or adolescent patients] Orthopade. 2016;45(12):1058-65. 